\title{
Philosophical Responses to Underdetermination in Science
}

\begin{abstract}
What attitude should we take toward a scientific theory when it competes with other scientific theories? This question elicited different answers from instrumentalists, logical positivists, constructive empiricists, scientific realists, holists, theory-ladenists, antidivisionists, falsificationists, and anarchists in the philosophy of science literature. I will summarize the diverse philosophical responses to the problem of underdetermination, and argue that there are different kinds of underdetermination, and that they should be kept apart from each other because they call for different responses.
\end{abstract}

Park, Seungbae (2009). "Philosophical Responses to Underdetermination in Science", Journal for General Philosophy of Science 40: 115-124.

Seungbae Park

Division of General Studies

Ulsan National Institute of Science and Technology

Republic of Korea

\section{Introduction}

The problem of underdetermination is one of the most widely discussed topics in the scientific realism-antirealism debate. The problem occurs, roughly, when a theory competes with rival theories. The rival theories make different claims about unobservables, but make (more or less) the same claims about observables. For example, von Neumann and Dirac's standard version of Copenhagen interpretation of quantum mechanics competes with Bohmian mechanics. Both theories make the same claims about macroscopic events, but different claims about microscopic events. According to the former, macroscopic events are determinate, microscopic events are probabilistic, and nothing travels faster than light. According to the latter, on the other hand, both macroscopic and microscopic events are determinate, but there exists something traveling faster than light. Now, the question arises as to which of the rival theories is true or the best. Different philosophical positions are available vis-à-vis the problem of underdetermination in general. I intend to summarize all of them in the literature and their shortcomings. Next, I shall classify underdetermination into different kinds, sketch different responses to each kind of underdetermination, and draw some philosophical morals from the different kinds of underdetermination. 


\section{Philosophical Reactions to Underdetermination in General}

Philosophers of different schools of thought have different reactions to the problem in which a scientific theory competes with its rival scientific theories. Instrumentalists' response is that a scientific theory is not a description about the world but an instrument for organizing thoughts about observables, i.e., connecting and relating observational data. So the theory is not capable of being true or false but capable of being useful or useless. Rival theories yielding the same predictions are not one and the same instrument but different instruments. So we are to choose one of them. We choose a simple theory over complex ones not because it is more likely to be true but because it is a more convenient instrument than the others. Now, an advantage of instrumentalism is that it takes less epistemic risk than scientific realism which recommends that we choose one of the rival theories and believe that it is true. Let me point out, however, that the advantage comes at a cost. Suppose that an instrumentalist puts forward a positive theory of his own in a social world in which every cognitive agent plays a fair game. His cognitive colleagues will in turn take the instrumentalist attitude toward it, refusing to believe that it is true. Thus, if the instrumentalist wants others to believe that his theory is true, he should be willing to believe that their theories are true.

Unlike instrumentalists, logical positivists believe that rival theories are one and the same theory. This surprising conclusion stems from their view of how we should understand the content of a scientific theory. For them, a scientific theory is a collection of theoretical and observational statements, and theoretical language is to be translated into observational language. The net result of the translation is that rival theories make the same claims about the world. Any rival theories making the same claims about observables are numerically identical with each other. Hence, there is no need to adjudicate among them. An epistemological worry is what ultimately motivates logical positivists to take the attitude that theoretical language is to be translated into observational language. In my view, a problem with the logical positivist attitude is that an initiator of a theory may have an intention that the theory be understood literally. Then, on what grounds can logical positivists claim that the theory is to be purged of theoretical language?

Unlike logical positivists, constructive empiricists literally interpret theories, asserting that theoretical language is not to be translated into observational language. Hence, rival theories are distinct from one another, making different claims about unobservable parts of the world. However, they agree with logical positivists that we should limit our beliefs on observables. The principle of economy enjoins us only to 
believe that the rival theories are empirically adequate. ${ }^{1}$ Theoretical virtues, such as simplicity and explanation, are not epistemic but pragmatic. It is not the case that a simple theory with high explanatory power is more likely to be true than a complex theory with low explanatory power. Put differently, empirical equivalence entails epistemic equivalence, and we do not have any means to know which of the empirical equivalents is true. My problem with constructive empiricists' reaction to the problem of underdetermination is that it is not clear why we should settle for empirical adequacy as opposed to approximate empirical adequacy or anything less than approximate empirical adequacy. ${ }^{2}$ After all, "there is a slide here from empirical equivalence to empirical adequacy" (Psillos, 1997: 370). Put differently, from the mere fact that rival theories produce similar predictions, it does not necessarily follow that they are empirically adequate. In fact, they may be far less than empirically adequate.

Like constructive empiricists, scientific realists believe that a scientific theory is to be literally understood. They believe, however, that we have means to know which of the rival theories is more likely to be true. Glymour, for example, claims that observations support theories discriminately.

My idea is that the body of evidence that distinct theories hold in common, the phenomena that both theories save, may nonetheless provide differing support for the two theories, more reason to believe one than the other, more confirmation of one than the other. Not because one theory is a priori more plausible or probable than the other but, roughly, because one theory is better tested than the other by the body of evidence in question. (Glymour, 1980: 342)

Laudan and Leplin also contend that the same observational evidence may yield different degrees of inductive support for different theories.

...the relative degree of evidential support for theories is not fixed by their empirical equivalence. (Laudan and Leplin, 1991: 460)

That is, a theory can be better confirmed than its rival theories, although it is empirically equivalent to them. Thus, for scientific realists, empirical equivalence does not entail epistemic equivalence.

Holists would argue that it is self-defeating that empirical equivalence entails

\footnotetext{
1 A theory is empirically adequate if and only if what it says about observables is true.

2 I propose that a theory is approximately empirically adequate just in case most of its observational
} 
epistemic equivalence. A theory characteristically issues in observational consequences only in conjunction with auxiliary theories and statements of initial conditions as Duhem (1905/1954) pointed out. The auxiliary theories should be assumed to be true for the main theory to compete with its rival theories. But it is illegitimate to assume that the auxiliary theories are true if empirical equivalence entails epistemic equivalence because they themselves are competing with their empirically equivalent rival theories. If there is no basis for preferring the auxiliary theories to their rival theories, they cannot be assumed to be true. Leplin puts the above point as follows:

What has not been recognized is that the truth of UD would prevent the determination that theories are empirically equivalent in the first place. (Leplin, 1997: 154-155)

It appears that the Quine-Duhem's thesis, according to which it is not a single theory but a group of theories that confront observations, can be utilized to combat the problem of underdetermination. I must point out, however, that Leplin's strategy works only for cases in which auxiliary theories are required to derive observational consequences from a main theory. In some cases, a main theory needs only statements of initial conditions to yield observational consequences. Poincare's example to be explained below fits this kind of underdetermination.

Theory-ladenists, in line with Gestalt psychology, could argue that the presupposition of the problem of underdetermination - competing theories are empirically equivalent - is false. The grounds for this provocative claim are that observation is theory-laden. Hence, scientists holding different theories in their minds have different observational beliefs. Since rival theories are not empirically equivalent, the question as to which of the empirically equivalent rival theories is true cannot even be raised. Thus, theory-ladenness undercuts one of the major weapons, the problem of underdetermination, scientific antirealists wield against scientific realism. In my view, however, scientific realists cannot invoke theory-ladenness to combat the problem of underdetermination. If there is no common observational ground between rival theories, it is not clear what the rational basis is for preferring one theory over other theories. Thus, theory-ladenness undermines not only the problem of underdetermination but also scientific realism. Now, in order to refute theory-laddenists' claim, we only need to point to a historical fact that Kepler borrowed observational data from Tycho (Kuhn, 1957: 206).

consequences are true. 
Brahe's skillful observations were even more important than his system in leading his contemporaries toward a new cosmology. They provided the essential basis for the work of Kepler, who converted Copernicus' innovation into the first really adequate solution of the problem of the planets. (Kuhn, 1957: 206)

The above example shows that the theory-ladenists are wrong to say that scientists of rival paradigms didn't share observational data in the history of science.

Antidivisionists would argue that the problem of underdetermination is saddled with the problematic distinction between observables and unobservables. In fact, Maxwell (1962: 9) argues that there is no principled distinction between observables and unobservables. We can see objects through windowpanes, glasses, telescopes, light microscopes and electron microscopes. It is clear that objects we see through windowpanes are observable. It is also clear that objects we see through electron microscopes are unobservable. But it is not clear where we should draw the line between observables and unobservables. Hence, it is not clear exactly what theories are underdetermined. Van Fraassen, however, would reply that since there are clear cases of underdetermination, the predicate 'underdetermination' is viable. After all, he says that "A vague predicate is usable provided it has clear cases and clear counter-cases" (1980: 16). Moreover, scientific realists cannot utilize Maxwell's move to solve the problem of underdetermination, for his argument from the continuum above also undercuts the realist view that true predictions confirm a scientific theory. Prediction statements are also on a continuum from observational to theoretical. Some of them are clearly theoretical. Consider the following example (Hung, 1997: 92): The nucleus of an atom may emit an electron known as a beta particle when it disintegrates. From this claim in conjunction with the principle of conservation of mass-energy, it deductively follows that the mass-energy of the original nucleus is equal to that of the remnant and the beta particle. This deductive consequence is obviously theoretical. Hence, further steps of deduction are required to arrive at observational consequences. In a nutshell, scientific realism and antirealism are in the same boat vis-à-vis the problem of the fuzzy distinction between observables and unobservables.

For falsificationists, we can never know which of rival theories is true. Humean skepticism about induction drives them to hold the view that true observational consequences are not capable of justifying a scientific theory, although false observational consequences are capable of falsifying it. Thus, empirical tests are not a method to discover a true theory but only a method to eliminate false theories. Since we 
cannot discover a true theory in the first place, we do not have a problem of deciding which of the rival theories is true. What we need to do when a theory competes with rival theories, according to the falsificationists, is to seek further observational consequences that would falsify all of them. Now, one of the well-known problems with falsificationism is that true observational consequences do not deductively prove that a scientific theory is false. As Duhem (1905/1954) notes, what confronts observations is not a single theory but a group of theories. So a conflict between the observations and the group of theories does not necessarily falsify a theory that is under a test. It might be other theories in the group that we should throw away as being false. Another serious flaw with falsificationism is that it is not clear how we could reconcile falsificationism with our practice of using a scientific theory to make future predictions, as Salmon points out:

In particular, positive instances do not provide confirmation or inductive support for any such unrefuted generalization. At this stage, I claim, we have no basis for rational prediction. (Salmon, 1981: 435)

If the problem of induction forbids true observational consequences from lending inductive support to a scientific theory, then impressive past performance of the theory in making true predictions should not be taken either to constitute an epistemic basis for believing that future predictions are true. Then, why do we use a scientific theory to make future predictions?

Anarchists would argue that when we are faced with an underdetermination we should not try to decide which of rival theories is true or false. Rather, we should come up with more alternative theories. In fact, Feyerabend claims that it is good to have many alternative theories.

You can be a good empiricist only if you are prepared to work with many alternative theories rather than with a single point of view and 'experience.' (Feyerabend, 1963: 923)

The justification for this view is that the invention of alternative theories leads to the discovery of new empirical facts and to a much sharper criticism of an accepted theory than does the mere comparison with observations. The point of criticizing the accepted theory is not to ascertain whether it is true or false but to avoid turning it into a dogma. In my view, however, this method for avoiding dogma comes at a price. If scientists had 
merely criticized an accepted theory by multiplying alternative theories, and if they had not adjudicated between them in the history of science, false theories, such as Ptolemaic theory and the caloric theory, would still compete with the Copernican theory and the kinetic theory today. Then, it is not clear that we can claim to have gained knowledge about unobservables. Multiplying rival theories seems to be beneficial on the realist point of view only when scientific research in an area is at its early stage of development. If the research becomes mature enough in that area, we might as well try to decide which of the rival theories is true. Otherwise, we will only flounder in permanent controversies over the nature of the unobservable parts of the world without gaining any knowledge about it. But scientific antirealists, since they are not interested in gaining theoretical knowledge at all, would welcome Feyerabend's recommendation that we invent rival theories without trying to decide which of them is true or false. Multiplying rival theories is good as long as it enhances our empirical knowledge.

So far I have summarized various philosophical reactions to the problem of underdetermination in general that are scattered around in the literature. In sketching the reactions, however, I did not rigorously formulate the problem of underdetermination. Underdetermination, it will be shown in the next section, can be classified into different kinds, depending on what the nature of rival theories is. The different kinds of underdetermination, I will argue, give rise to different problems, calling for different answers.

\section{Different Kinds of Underdetermination and Reactions to Them}

\subsection{Permanent Underdetermination vs. Transient Underdetermination}

A theory is permanently underdetermined just in case it is one of empirically equivalent rival theories. The empirical equivalence implies that even advanced future evidence may not break the tie between the rival theories. Poincare (1902/1952) provides a useful example to illustrate this kind of underdetermination. Imagine a world of a finite sphere whose space is Euclidean. Let $R$ be its radius, and $r$ the distance of any point from the center. The temperature of the sphere varies in accordance with the formula: $R^{2}-r^{2}$. As a result, rigid rods expand as they move toward the center of the sphere, and shrink as they move toward the circumference of the sphere. Light travels on circular paths in this world. The refractive index of the light medium is given by the formula: $1 /\left(R^{2}-r^{2}\right)$. Now, the world described so far by the Euclidean framework can also be described by the non-Euclidean framework. The non-Euclidean story holds that rigid rods stay the same size when moved around, light travels in straight lines, the world is infinite, and the space is Lobachevskian. The Euclidean theory and the non-Euclidean theory can equally 
cope with all the present and future observational data the denizens of the world could come up with. So the theories are under permanent underdetermination.

The idea of permanent underdetermination is saddled with a problematic assumption that the distinction between observables and unobservables is rigid. Laudan and Leplin point out that with technological advances we will have more accurate and extensive data that will break the tie between the rival theories.

This result already shows that findings of empirical equivalence are not reliably projectable, since we cannot reliably anticipate which of a theory's now unobservable consequences may become observable. (Laudan and Lepline, 1991: 453)

Maxwell further points out that our descendents, as a result of genetic mutations that might occur in the future, may be able to observe what we cannot observe now.

Suppose that a human mutant is born who is able to "observe" ultraviolet radiation, or even $\mathrm{X}$ rays, in the same way we "observe" visible light. (Maxwell, 1962: 11)

Let me add that evolution has been enhancing the capabilities of our sensory organs. We can now observe things a single-celled organism could not observe billions of years ago. So it is "likely" that our descendents will be able to observe what we cannot observe now.

Permanent underdetermination contrasts with what Sklar (1975: 380) calls transient underdetermination. A theory is transiently underdetermined if and only if it is one of the rival theories which make different claims about unobservables but are compatible with all the evidence available at a point of time in the development of science. Note that future evidence may break the tie between the rival theories in such underdetermination. In this sense, the Copernican theory was under transient underdetermination, when it competed with the Ptolemaic theory. The two theories were not empirically equivalent. They made slightly different claims about observables. For instance, the Copernican theory predicted the phase of Venus but the Ptolemaic theory did not. But the scientific community back then did not have a telescope to observe the phase of Venus. I must point out that transient underdetermination poses no less threat to scientific realism than permanent underdetermination at the point of time when all the available evidence is compatible with rival theories. After all, at such a point of time we 
have no choice but to appeal to such theoretical virtues as simplicity and explanatory power in order to determine which of the rivals is the best. But these virtues are exactly the ones we have to appeal to when theories are permanently underdetermined.

\subsection{Moderate Underdetermination vs. Radical Underdetermination}

A theory is moderately underdetermined just in case it is one of rival theories that make slightly different claims about unobservables. Suppose that Newton's theory of motion is exactly true, and that it consists of the three laws of motion, the law of gravity, and the hypothesis that the center of the gravity of the solar system is at rest with respect to absolute space. A rival theory, having the three laws of motion and the law of gravity as its components, claims that the center of gravity moves, say, $10 \mathrm{~km} / \mathrm{h}$ with respect to absolute space. We can generate infinitely many rival theories by varying the absolute velocity of the center of the gravity. The rival theories are all observationally equivalent but incompatible with each other. Now, suppose that scientists were not confident of the absolute velocity of the center of the gravity of the solar system. Then, the rival theories, I contend, are all approximately true ${ }^{3}$ because they share the important assumptions: the three laws of motion and the law of gravity. The philosophical significance of moderate underdetermination is that it is not a serious threat to scientific realism once scientific realists settle for approximate truth as opposed to approximate truth.

Moderate underdetermination contrasts with what I call radical underdetermination. A theory is radically underdetermined when it is one of the rival theories that make radically different claims about unobservables. The claims are so radically different from each other that even if one of the theories is true, the rest of them are not even approximately true. What can scientific realists say about this kind of underdetermination? Suppose that we have two groups of rival theories from two neighboring domains. One group of rival theories, making radically different claims about unobservables, competes with each other in Domain 1. The other group of rival theories, also making radically different claims about unobservables, competes with each other in Domain 2. But miraculously T1 in Domain 1 coheres with T1' in Domain 2 , i.e., they hang together in such a way that an inference from $\mathrm{T} 1$ to $\mathrm{T} 1$ ' and vice versa is to some degree truth-preserving. What would explain the coherence of the two theories? Do they cohere with each other because they are (approximately) empirically adequate or because they are (approximately) true? The (approximate) empirical adequacy is not robust enough to make the two theories cohere with each other. After all, what the theories say about observables is only tenuously connected with what they say

\footnotetext{
${ }^{3}$ Defining 'approximate truth' is such a huge issue that I set it aside here.
} 
about unobservables if they are radically underdetermined. Only the (approximate) truth has the force to make the two theories cohere with each other. Here is an analogy to illuminate this abstract point. We cannot hold together two lizards which do not get along with each other by tying their tails together. They will just run away by cutting their tails off from their main bodies. The only way to hold them together is to tie their main bodies together. In actual science, some theories of different domains do cohere with each other. The theory of evolution coheres with the theory of DNA and with the theory of plate tectonics. So it is false that such theories are under radical underdetermination.

\subsection{Actual Underdetermination vs. Possible Underdetermination}

A theory is actually underdetermined if and only if it competes with rival theories we conceived of. In this sense, von Neumann and Dirac's standard version of Copenhagen interpretation of quantum mechanics is under actual underdetermination, for it competes with Bohemian mechanics which we conceived of. Concerning the actual underdetermination, Psillos contends that it is only a local phenomenon, not a global phenomenon, in science.

More generally, the existence of empirically equivalent theories can create a genuine problem only if it is shown to be a global phenomenon. This is what UTE should demonstrate if it is to ditch any hope of discovering the blueprint of the universe. But, there is no relevant evidence for this. (Psillos, 1999: 168)

The presence of some rival theories in a particular domain does not support the sweeping generalization that there are rival theories in all domains. Specifically, from the fact that there are some rival theories to physical theories it does not follow that there are serious rival theories to nonphysical theories, such as biological theories and geological theories. I must add that nonphysical theories are neutral as to which of the rival physical theories is true. The molecular structure of water, for instance, is neutral as to whether space is Euclidean or non-Euclidean. So even if we take an antirealist attitude toward the geometrical structure of the universe, it does not follow that we should also take the same attitude toward, say, DNA.

Actual underdetermination is in contrast with what I call possible underdetermination. A theory is possibly underdetermined if and only if it competes with rival theories we have not yet conceived of. In this sense, all current scientific theories are under possible underdetermination. Now, what follows from possible 
underdetermination? Van Fraassen argues that in order to believe that a theory is likely to be true, we must first prove that truth is likely to lie in the range of the conceived theories rather than in the range of unconceived theories.

So our selection may well be the best of a bad lot... For me to take it that the best of set $\mathrm{X}$ will be more likely to be true than not, requires a prior belief that the truth is already more likely to be found in X, than not. (van Fraassen, 1989: 143)

My reply to this claim is that a normative statement does not follow from a mere possibility statement. It is possible, for instance, that I am not a human being but a pig dreaming that I am a human being. Does it follow that I should run away from a butcher upon seeing him on the street? In my view, a normative statement follows from a statement with significant probability. If there is significant probability, for instance, that a huge meteorite will fall in New York tomorrow, then New Yorkers should evacuate the area. Likewise, scientific realists have the burden van Fraassen thinks they do only when there is a significant probability that truth is in the range of unconceived theories. But what is the reason for thinking that there is such a probability?

\subsection{Regular Underdetermination vs. Bizarre Underdetermination}

A theory is under regular underdetermination when it competes with regular scientific theories. All the scientific theories that competed with each other in the history of science were under regular underdetermination. This kind of underdetermination contrasts with bizarre underdetermination. A theory is under bizarre underdetermination if and only if it competes with bizarre theories. The bizarre theories are generated by the following algorithms:

For any theory $\mathrm{T}$, construct the theory $\mathrm{T} 1 \mathrm{which}$ asserts that the empirical consequences of $\mathrm{T}$ are true, but that none of its theoretical entities exist. (Kukla, 2001: 21-22)

Given theory $\mathrm{T}$, construct $\mathrm{T} 2$ which asserts that $\mathrm{T}$ holds when somebody is observing something, but that when there's no observation going on, the universe follows the laws of some other theory T'. (ibid: 23)

Kukla claims that scientists do not bother to give arguments against these theories, and 
that scientists take bizarre underdetermination as giving rise to a philosophical problem, not a scientific problem.

Kukla's response to the problem of bizarre underdetermination is to argue that the bizarre theories have the prior probability of zero. Hence, it is rational for scientists to disregard them. A problem with the response is that it is not clear why bizarre theories, not regular theories, have such a probability. My alternative answer to the problem is that the algorithms have the potential to foster Cartesian skepticism. What is the reason for thinking that the algorithms have the potential to produce Cartesian skepticism? Just replace 'theory' with 'perceptual belief' in the above algorithms. The net results are as follows:

For any perceptual belief B, construct the perceptual belief B1 which asserts that we have all the relevant perceptions, but the external world does not exist.

Given perceptual belief B, construct B2 which asserts that B holds when somebody is making an observation, but that when there's no observation going on, the universe conforms to other perceptual belief B'.

Note that these two algorithms generate skepticism about perceptual beliefs. Now, what is wrong with Cartesian skepticism? As Maxwell (1998) points out, we cannot make sense of scientific enterprise in terms of Cartesian skepticism. If Maxwell is right, it is rational for scientists to disregard the algorithmically generated theories.

\section{Conclusion}

I have surveyed major philosophical positions on the topic of underdeterminiation in the literature. Inevitably, my own views have protruded here and there. In closing, I wish to draw a conclusion from the classification I made of underdetermination. The different kinds of underdetermination should not be conflated for a couple of reasons. First, scientific realism is vulnerable to some kinds of underdetermination: permanent underdetermination, transient underdetermination, radical underdetermination, actual underdetermination, and regular underdetermination. But it is not so susceptible to other

kinds of underdetermination: moderate underdetermination, possible underdetermination, and bizarre underdetermination.

Second, a reaction that is appropriate to one kind of underdetermination may not be appropriate to another kind. Suppose that T1 competes with T2, and that they make only slightly different claims about unobservables. That is, they are under moderate 
underdetermination. Suppose also that scientific realists believe that $\mathrm{T} 1$ is approximately true, and that scientific antirealists believe that it is empirically adequate. Then, it would be inappropriate for the scientific antirealists to claim that their position is epistemically safer than that of the scientific realists. After all, it is not clear that approximate truth is harder to come by than empirical adequacy when a theory is under moderate underdetermination.

\section{References}

Duhem, P. (1905), The Aim and Structure of Physical Theory. trans. P. Wiener, Princeton, NJ: Princeton University Press (1954).

Feyerabend, P. (1963), "How to Be a Good Empiricist - A Plea for Tolerance in Matters Epistemological", in Philosophy of Science, The Delaware Seminar. vol. 2: 3-39. In Curd M. and J. Cover (eds) (1998), Philosophy of Science: The Central Issues, New York: W. W. Norton \& Company, Inc.

Glymour, C. (1980), Theory and Evidence. Princeton, NJ: Princeton University Press.

Hung, E. (1997), The Nature of Science: Problems and Perspectives. Belmont, CA: Wadsworth Publishing Company.

Kuhn, T. (1957), The Copernican Revolution. Cambridge: Harvard University Press.

Kukla, A. (2001), "Theoreticity, Underdetermination, and the Disregard for Bizarre Scientific Hypothesis", Philosophy of Science 68: 21-35.

Laudan, L. and Leplin, J. (1991), "Empirical Equivalence and Underdetermination", Journal of Philosophy 48: 449-472.

Leplin, J. (1997), A Novel Defense of Scientific Realism. New York: Oxford University Press.

Maxwell, G. (1962), "The Ontological Status of Theoretical Entities", in Minnesota Studies in the Philosophy of Science III. Minneapolis, MN: University of 
Minnesota Press.

Maxwell, N. (1998), The Comprehensibility of the Universe: A New Conception of Science. Oxford: Claredon Press.

Poincare, H. (1952), Science and Hypothesis. Translated by Greenstreet, W., New York: Dover Publications, inc. (original French edition published in 1902).

Psillos, S. (1997), “How Not to Defend Constructive Empiricism: A Rejoinder”, The Philosophical Quarterly 47: 369-372.

Psillos, S. (1999), Scientific Realism: How science tracks truth. New York: Routledge.

Salmon, W. (1981), "Rational Prediction", British Journal for the Philosophy of Science 32: 115-125. In Curd M. and J. Cover (eds) (1998), Philosophy of Science: The Central Issues, New York: W. W. Norton \& Company, Inc.

Sklar, L. (1975), "Methodological Conservatism", Philosophical Review 84: 374-400.

van Fraassen, B. (1980), The Scientific Image. Oxford: Oxford University Press. (1989), Laws and Symmetry. Oxford: Oxford University Press. 